\title{
Development and Flight Test of a Reconfigurable Avionics Research Pod for the USAF Test Pilot School
}

\author{
Adam MacDonald $^{1}$, Michael J. Shepherd ${ }^{2}$ \\ USAF Test Pilot School \\ 220 South Wolfe Ave. \\ Edwards Air Force Base, CA 93524 \\ $661-277-2125^{1}, 661-277-6551^{2}$ \\ adam.macdonald@edwards.af.mil ${ }^{1}$, michael.shepherd@edwards.af.mil ${ }^{2}$
}

\begin{abstract}
Considerable time and money are spent on the modification of fleet-support test aircraft in order to enable carriage of novel research experiments that require in-flight test and demonstration. In many cases, the cost of aircraft modification exceeds the cost of the flight test hardware and flight hours. The USAF Test Pilot School (TPS) has worked closely with the Air Force Institute of Technology (AFIT) to develop and flight test the Reconfigurable Airborne Sensor, Communication and Laser (RASCAL) pod. The pod concept will revolutionize the way that USAF TPS conducts Test Management Project training, and will enable rapid transition of cutting-edge technology under development at AFIT and national laboratories to the demanding airborne flight environment. The development and flight test of RASCAL are discussed, as are future concepts of operation expected to be conducted at the USAF TPS.
\end{abstract}

\section{TABLE OF CONTENTS}

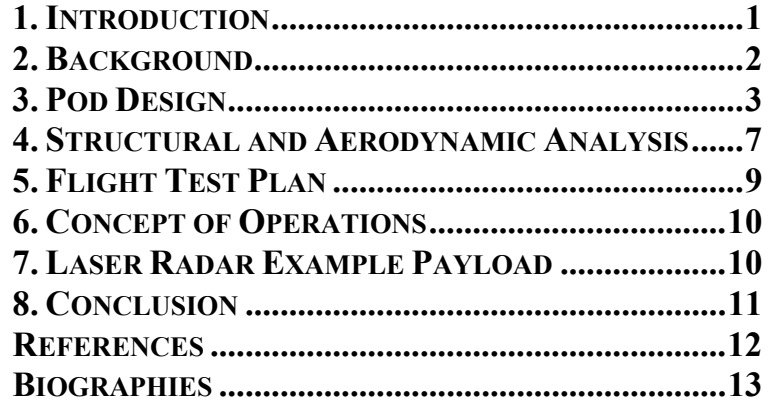

\section{INTRODUCTION}

The US Air Force Test Pilot School (TPS) [1] is charged with the mission of producing highly adaptive, critical thinking, full-spectrum flight test professionals. To meet this challenge, the year-long flight test course equips approximately 40 Air Force, Navy, Marine, various foreign national officers and select civilians with the tools necessary

\footnotetext{
1

${ }^{1}$ U.S. Government work not protected by U.S. copyright.

${ }^{2}$ IEEEAC paper \#1017, Version 2, Updated Jan 6, 2008
}

to plan, execute and report on virtually all aspects of flight test. The TPS curriculum consists of academic and flight instruction in the fields of aircraft performance, flying qualities, systems and test management. The test management curriculum culminates in a capstone, realworld flight test project [2]. In a given academic year, eight distinct such capstone test projects are conducted using resources drawn primarily from the Air Force Flight Test Center [3], co-located with TPS at Edwards Air Force Base, in the Mojave Desert of California.

In contrast to the rather scripted flight test missions flown during other portions of the TPS curriculum, these test management projects (TMP) challenge students with realworld test of cutting-edge airborne technology. Of these eight annual TMPs, six are typically fed by students who gain their masters degree in either aeronautical or electrical engineering through a joint AFIT/TPS program. These six students attend the Air Force Institute of Technology (AFIT) [4] at Wright Patterson AFB, near Dayton, Ohio for a period of 15 months, and then transition to TPS for class start dates in either January or July. During the latter portion of their education at AFIT, these select students develop novel hardware and software projects to be introduced into the flight test environment approximately a year later, as they enter the final phase of their education at TPS. Upon graduation from TPS and successful defense of their flight test oriented thesis work, these students are granted a Master of Science in either aeronautical or electrical engineering. There exist easier paths to obtaining these degrees, however, it is our experience that these joint graduates provide a great deal of technical leadership to the flight test community as they progress through their careers in the Air Force as well as industry.

Unfortunately, the novel technology developed by these joint program students tends to be difficult to incorporate onto test aircraft. This is simply the nature of the beast. Modifications to fleet aircraft, even dedicated flight test airframes, is a laborious and expensive process - and for good reason. Unproven hardware and software carries with 
it the potential to disrupt delicate on-board flight-critical systems. Air Force regulations have been carefully crafted to ameliorate the deleterious effects of incompatible flight test hardware. Compliance with these regulations is a costly, time-consuming practice; required modifications often take weeks if not months and many tens of thousands of dollars. Aside from the financial burden of such tests, the assigned test aircraft is unavailable for other missions during the modification process period.

This paper is organized as follows. A brief background of past flight test management projects conduced at the USAF TPS is offered. The reconfigurable pod concept is introduced in the context of the academic and research environment that surrounds the joint AFIT/TPS program. The design of the reconfigurable pod is addressed, with special attention to the requirements of aeronautical and structural analysis. The planned flight test is outlined and discussed, followed by a brief description of the planned operational concept. Finally, an example payload is discussed, along with the research and academic benefits of such an initial flight test arrangement.

\section{BACKGROUND}

The quest for a reconfigurable flight test platform was launched in the early months of 2006 [5], when the number of Joint AFIT/TPS masters degree students increased by 100 percent from three to six joint students per year. Although several joint projects would continue to require significant modifications of flight-test aircraft, a large number of these projects would have the capability of flying within a modular structure that could be mounted externally on any suitable fleet-support aircraft. Such a concept would obviate the need to specially modify a particular aircraft and thus avoid the associated schedule and budget risks. To illustrate the utility of such a "podular" path to recurring flight test, several past flight test management projects are reviewed.

The fall 2007 joint AFIT/TPS project GOOD LOOKIN' [6] explored the ability to enhance navigation accuracy of a low-cost, high-drift rate micro electro-mechanical system (MEMS) inertial measurement unit (IMU) by observation of random features of interest observed on the ground. Although navigational enhancement is routinely performed by fusing positional and rate information gathered from global positioning system (GPS) receivers, the DoD and other agencies are interested in the capacity to maintain precision navigation during periods of GPS non-availability. Furthermore, navigation in dense urban environments and within caves presents a challenging problem for future autonomous flight operations.

Figure 1 shows the implementation of project GOOD LOOKIN' on an AFFTC fleet support C-12C (Beechcraft
King Air variant) aircraft. A specially modified optical port located under the co-pilot seat was fabricated to house the cameras and other optical support equipment. The relatively large interior of the C-12 aircraft provided space to house the processing and display equipment required for collection and real-time analysis of the optical data collected in flight. Although the project was regarded as quite successful, several limitations were imposed by the C12 carriage platform. Most notably, the restricted operating airspeed and dive angle limitations of the $\mathrm{C}-12$ prevented collection of ballistic (free-fall) weapon profiles, typical of munitions released from delivery aircraft en-route to a tactical target. In addition, the modified optical port disabled the ability to pressurize the cockpit and aircraft interior, requiring relatively low-altitude operation. Finally, the necessary location of the optical port beneath the copilot seat severely restricted the size and configuration of the optical equipment used to conduct these experiments. Despite these technical restrictions, perhaps the most significant issue encountered during this project was the cost and time required to execute the modification to the $\mathrm{C}$ 12. The total dollar cost for this project was approximately $\$ 60,000$, and required the $\mathrm{C}-12$ be taken off the flight-line for two full weeks. Given the total fleet size of C-12s at the AFFTC is only four aircraft, a $25 \%$ reduction in fleet size for such a period of time is usually problematic. Note that these aircraft are shared by the TPS with other test squadrons on base, where they are shared between curriculum, test management projects, test, and test support sorties.

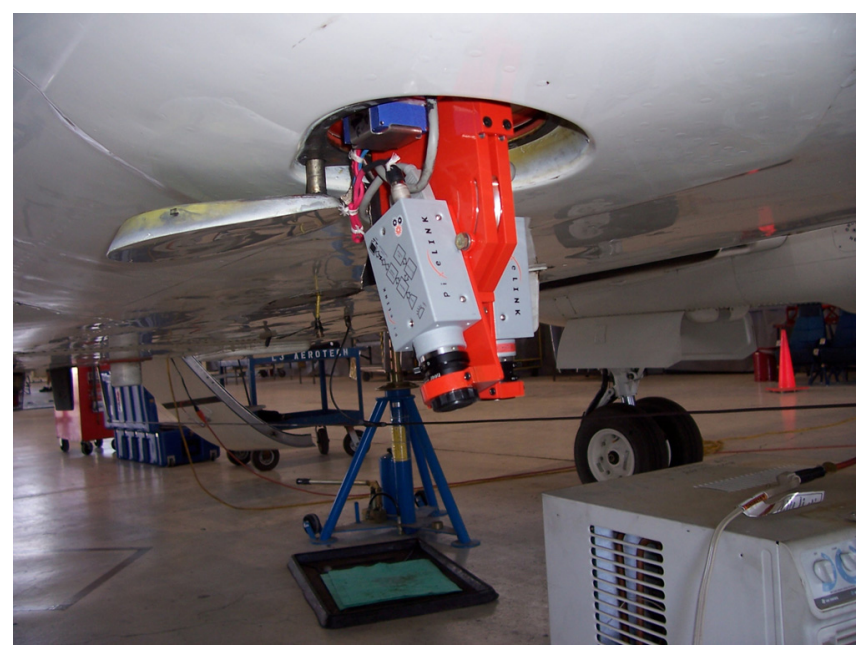

Figure 1 - External camera modification to AFFTC fleet support C-12C in support of project GOOD LOOKIN'. No protective dome was fabricated in order to simplify the project and reduce costs. Limited space beneath the co-pilot seat necessitated protrusion of a large portion of the camera equipment.

Project PEEPING TALON, conducted in 2003, served similar test and research objectives as GOOD LOOKIN', but provided a plethora of research data used in several 
research efforts in the field of image-aided inertial navigation [7], [8]. The test platform was a T-38 Talon aircraft, designed as a high-performance, supersonic jet trainer and used extensively for curriculum execution at the TPS. A gated-intensified CCD imaging camera was mounted to image through the side of the rear cockpit. Images were collected and time-tagged using a GPS timing reference to within 1 millisecond accuracy. Real-time video feedback of the collected imagery was displayed to the pilots by way of a liquid-crystal display (LCD) imaging device mounted in the cockpit. The imagery was ported to a video recorder for off-board analysis. The sideward camera mount allowed for ground target or stellar imaging as a function of aircraft bank angle. Figure 2 graphically depicts the locations of the major test item components.

Although the equipment used in PEEPING TALON was not unusual in terms of scientific complexity, the modification of the T-38 required significant engineering drawings and machining. In particular, the wiring and interconnect of the camera, GPS and recorder was formidable. The total time and expense for this particular modification was $\$ 90,000$ and three weeks of aircraft non-availability.

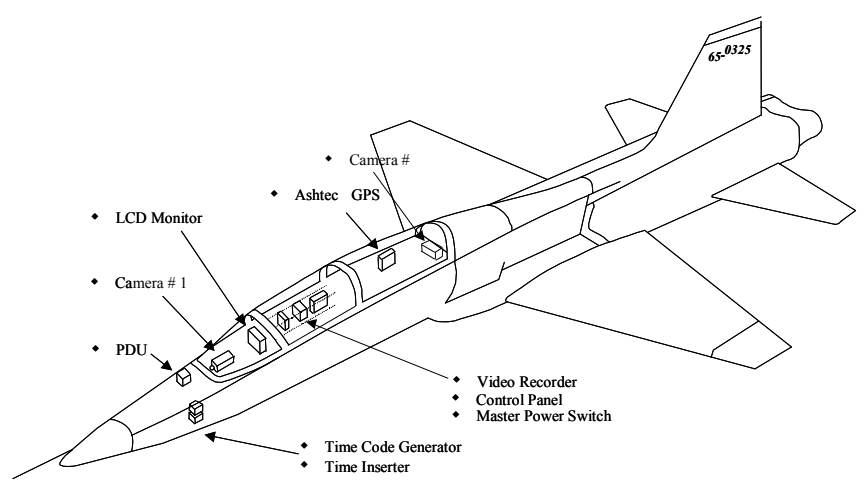

Figure 2 - Project PEEPING TALON equipment modification layout for fleet support T-38 aircraft.

Although scores of test management projects have been conducted over the past seventeen years of flight research via the joint AFIT/TPS process, a final example will serve to drive home the utility of a reconfigurable flight-test pod.

In response to the war-fighting training command, AFIT and the USAF TPS were asked to develop and test a system to expose fighter type aircraft to a realistic GPS jamming environment in order to enable development of effective counter-tactics [9]. Unfortunately, even limited area GPS jamming is extremely restricted due to the widespread proliferation of civilian GPS devices. To that end, a prototype system was developed to inject very low level RF noise directly into the aircraft GPS. Figure 3 shows the installation of the noise injection processor on an AFFTC fleet support T-38C.

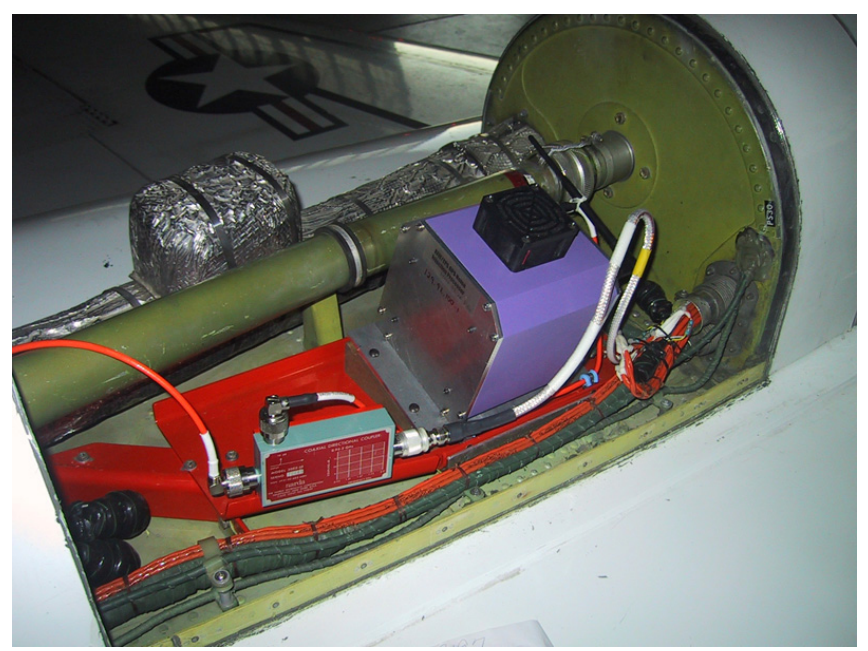

Figure 3 - GPS noise injection processor located in the upper spine of a fleet support T-38C. Access to this test equipment was made difficult by the large number of fasteners required to remove the spine access panel.

Due to the integrated nature of the RF noise injection procedure, a fairly complex control system was developed to enable, disable, modulate and sequence the noise source. The modification of the aircraft as required to route the necessary control cables was found to be cost prohibitive given the limited scope of the project. Such a modification was estimated to cost $\$ 50,000$, and require two weeks of aircraft down-time. Although the test team was able to execute the test for about half this cost by miniaturizing the components and installing them into a small void in the spine of the T-38C aircraft, the test was limited by the size of the processor and the limited wireless control mechanisms that were available. Hosting such a system on the RASCAL pod would have dramatically increased the system capability, and added a significant level of operational confidence during flight test execution.

The above examples demonstrate the need for a reduction in modification costs, both in terms of dollars and time spent in modification. Ever tightening budgets and workflow timelines require that innovative solutions are explored to reduce the number of modified fleet support aircraft while maintaining a high degree of agility and flexibility when introducing novel technology to the flight test environment.

\section{Pod DeSIGN}

\section{General Concept}

The reconfigurable airborne sensor, communication and laser pod (RASCAL) is based on the widely deployed SUU-20/A training bomb and rocket dispenser. Figure 4 shows the SUU-20/A under the wing of an AFFTC fleet 
support F-16B prior to execution of a weapons delivery test training mission. The basic SUU-20/A is approximately 10 feet long, 19 inches in width, and 12 inches high. It has an open bottom area where mechanical claws carry six inert practice bomblets, and four rocket tubes that span the length of the pod.

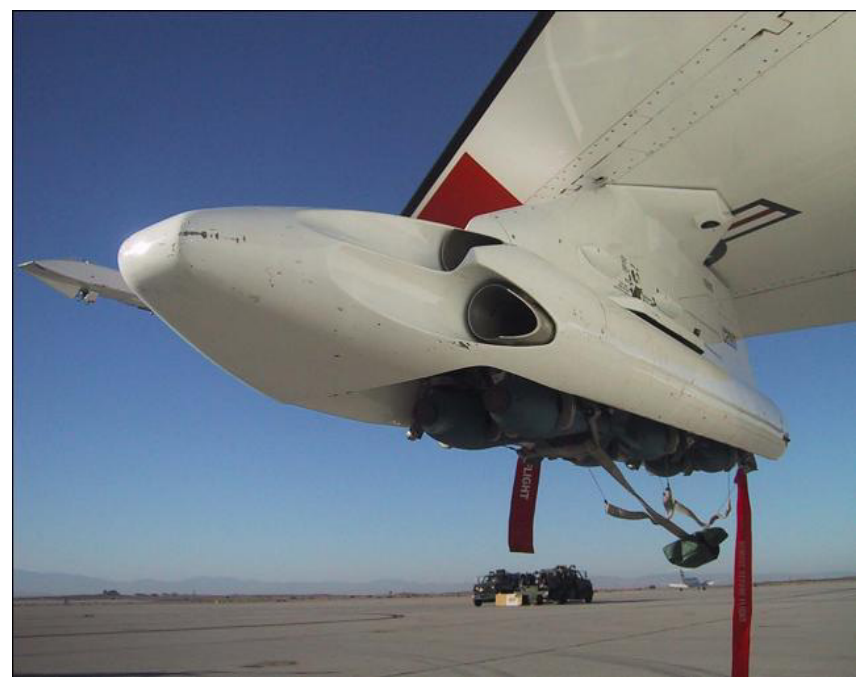

Figure 4 - SUU-20/A training bomb and rocket dispenser pod under right wing of AFFTC fleet support F-16B aircraft. The six blue egg-shaped devices are inert practice bomblets held by solenoid actuated claws. The four hollow rocket tubes are seldom used on Air Force training missions.

The SUU-20/A was chosen as a base for modification due to its wide variety of certified carriage aircraft. In addition to all variants of the F-16, the SUU-20/A is widely carried on AT-38B training aircraft, as well as all variants of the F15 Eagle. The importance of multi-aircraft carriage of the modified RASCAL cannot be overstated; any process that adds resource flexibility dramatically reduces test program risk. This is especially critical given the test program time constraints imposed by a busy TPS curriculum.

While the basic configuration of the SUU-20/A provided an adequate modification platform, its stock configuration lacked some basic features required of a reconfigurable avionics pod testbed. For example, the undercarriage lacked protection from the outside environment, requiring the addition of hinged doors. In addition, the inner width of the SUU-20/A was quite narrow, and needed to be widened considerably to allow up to 12 lateral inches of equipment to be installed.

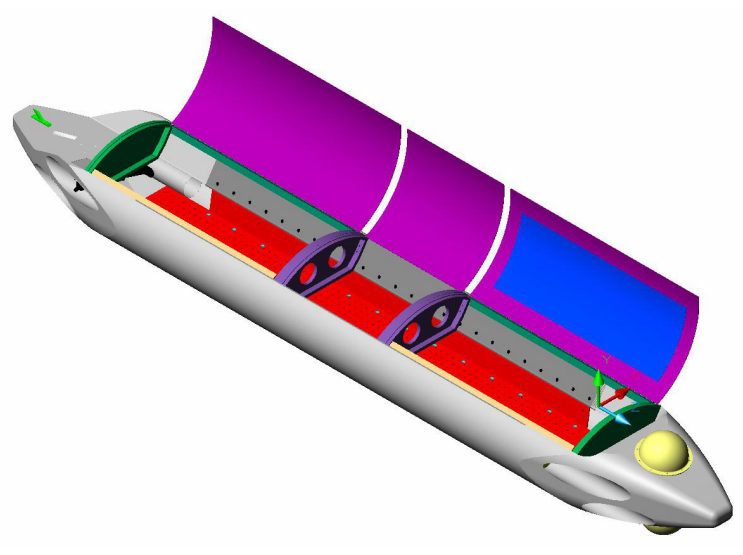

Figure 5 - Computer aided design rendering of modified SUU-20/A pod. Hinged doors provide easy access to forward and aft equipment bays. Semi-spherical optical domes on the forward top and bottom, as well as a large window in the forward bay provide camera access to the flight environment. Not shown is the permanently installed battery and charging system in the central equipment bay.

\section{Pod Structure Modification}

To provide structure to the pod and allow construction and mounting of three separate doors, milled aluminum truss structures were fabricated to span the width of the pod at various locations, yet provide space beneath to run cables and optical equipment. These were designed to provide additional stiffness to the structure to account for the material that was removed during the widening of the inner portion of the pod.

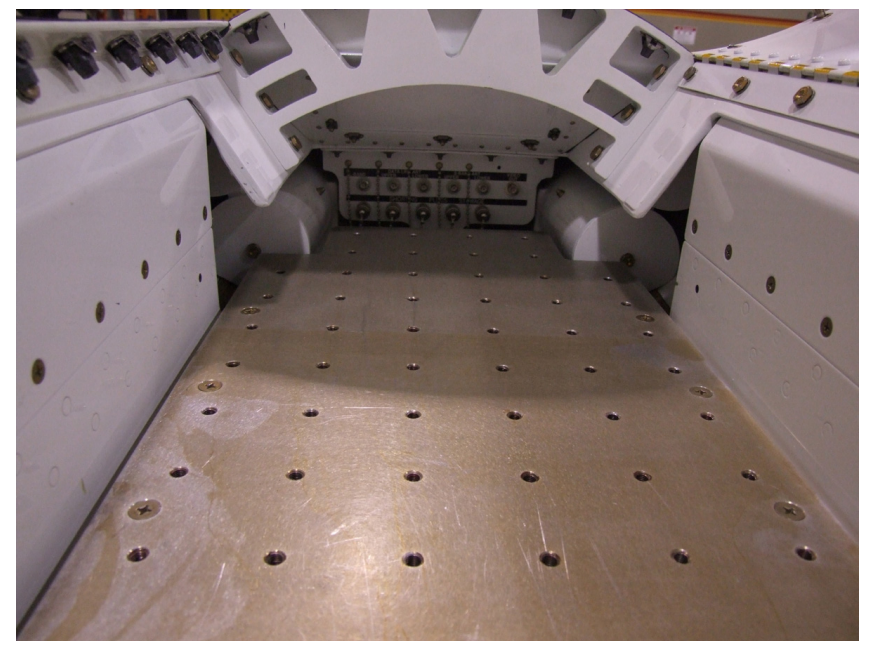

Figure 6 - Aft equipment bay breadboard. The mounting holes are drilled 2 inch on-center for simplified rigid mounting of various types of experimental equipment. It is expected that much of the aft bay space will be occupied by the PXI card cage for signal and data processing. 
In order to mount various unspecified test equipment components, a rigid, half-inch aluminum plate was fabricated to provide the mounting surface in each of the three equipment bays. This plate consists of a series of 2inch on-center $1 / 4$ "-20 mounting holes to provide easy beddown of equipment, and will provide a rugged, relatively non-flexing backbone for optical and electronic experiments. See Figure 6 for reference.

\section{Optical Sensor Access}

To provide ample opportunity to visually sample the surrounding environment, several optical ports were designed and integrated into the RASCAL. The prominent "bubbles" located on the upper and lower portions of the nose of the pod are six inch diameter clear polycarbonate domes positioned to allow surveying of forward portions of the environment. Figure 7 Shows these windows clearly. Small mirror systems or articulated cameras may be mounted on gimbals within these domes to provide a fairly large field-of-regard. Additionally, a large 10 by 14 inch, $1 / 4$-inch thick polycarbonate window is integrated into the forward equipment bay door. Figure 8 illustrates this large window.

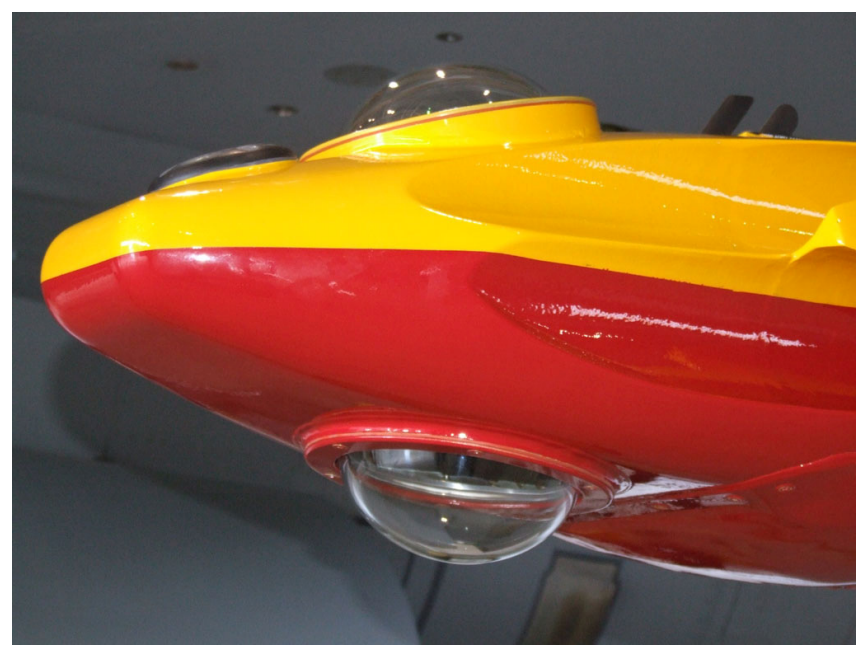

Figure 7 - Front section of the RASCAL clearly showing the hemispherical dome optical windows.

This large window provides an excellent, unobstructed view of the areas below the aircraft up to angles more than approximately 20 degrees below the aircraft waterline. A future expansion capability is provided by the four hollow rocket tubes that were designed into the baseline SUU20/A. Rather than fairing over these orifices, the holes were plugged and sealed. Simple removal and replacement of these plugs will allow for future installation of up to eight forward and aft facing cameras or laser emitters. Although the rocket tube orifices provide a relatively narrow field-of- regard, they provide an important opportunity to implement direct forward-facing cameras and illuminators.

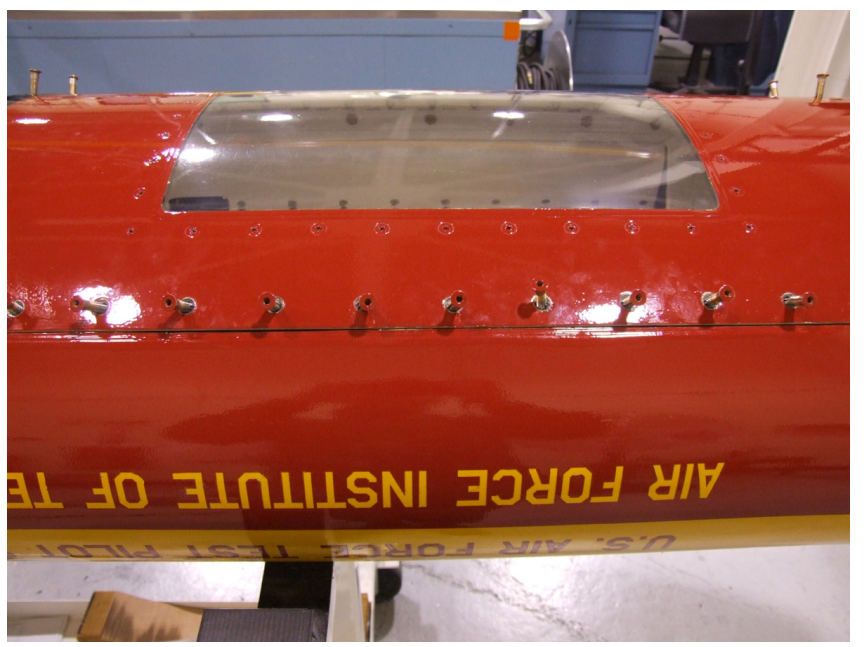

Figure 8 - Large polycarbonate window for downward survey of targets. Captive fasteners and hinged doors allow rapid access to equipment in the lab and on the flight-line.

\section{Power Subsystem}

A constant source of concern involved in flight test equipment installation is the availability of a clean, uninterrupted power source. Many flight test programs have been set back by the late acknowledgement that poor assumptions were made regarding the use of aircraft mains power. To this end, the RASCAL was designed to be relatively self-contained with regards to its power supply system. The center equipment bay is dedicated to a 24 Volt DC power storage and charging system. Four 18 Amp-hour 12 Volt sealed lead-acid batteries provide 36 Amp-hours of 24 Volt power to test equipment.

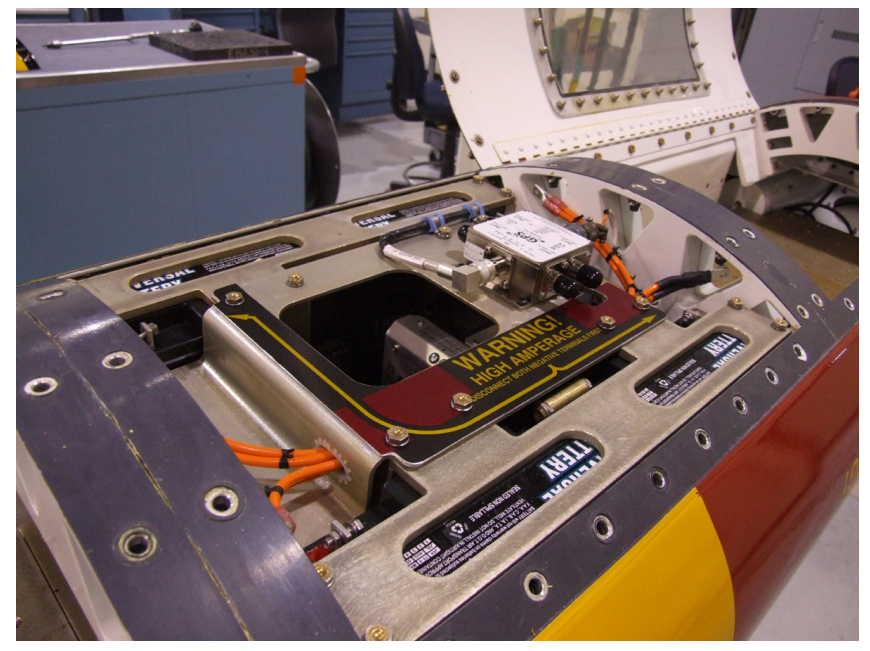

Figure 9 - Center section of the RASCAL pod which houses the four 18 Amp-hour sealed 12 Volt lead-acid batteries, together with high current charging system 
and power distribution panels. A GPS signal splitter atop the battery compartment allows amplified distribution of GPS signals to various instrumentation.

If necessary, the DC power may be inverted for $\mathrm{AC}$ powered equipment; however, this inversion process must be included separately with the test equipment. Typical operation will include pre-flight test equipment checkout without aircraft power, both in the laboratory, as well as on the flightline. During periods of availability of aircraft power, the batteries may be continuously charged using 115 Volt AC or 28 Volt DC aircraft power. The capacitive effect of the batteries and associated charger will dramatically reduce the ill effects of power surges and brownouts/blackouts experienced during aircraft engine start and generator cycling. Figure 9 illustrates the implementation of the battery and charging system.

\section{Rapid-Access Maintenance and Preflight}

The hinged door system allows rapid access to the forward and aft equipment bays by use of captive fasteners that reduce the possibility of foreign object damage to aircraft engines. Although the central door covering the battery is removable, it is not hinged as it covers a structural mounting point and flight-line access requirements are anticipated to be low.

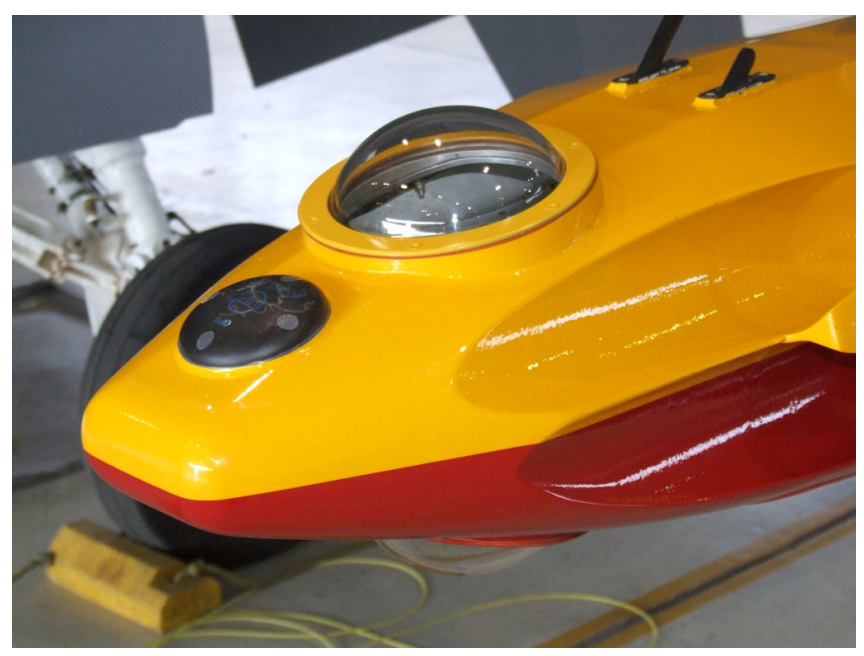

Figure 10 - Upper view of nose section showing 900 MHz and S-band upper antennas aft of hemispheirical dome, as well as the GPS antenna puck forward of the dome.

\section{RF Antennas}

The anticipated uses of a reconfigurable test pod include recurring radio-frequency $(\mathrm{RF})$ transmission and reception experiments. It is expected that the vast majority of experiments will require GPS constellation reception. A single GPS antenna is mounted on the upper forward deck of the pod. This location provides fairly good up-look capability while mounted on the F-16 carriage platform.
However, belly-mounting of the pod on an AT-38B will severely limit this up-look capability. In this case, GPS transmissions will be routed from the aircraft GPS antenna through pre-existing cables via the pylon of all AFFTC AT-38B aircraft. GPS signals are split into four amplified branches for use by timecode, navigation and other equipment requiring GPS signals.

Additionally, L-band, S-band and $900 \mathrm{MHz}$ antennas are mounted on the lower aft deck of the RASCAL. These antennas may be used for real-time parameter telemetry to a ground-control station. The S-band antenna possesses sufficient VSWR bandwidth to allow transmission of Wi-Fi data on the $2.4 \mathrm{GHz}$ Industrial, Scientific and Measurement (ISM) band. An additional S-band antenna is located on the top of the RASCAL upper deck to facilitate interpod communication using S-band equipment such as $2.3 \mathrm{GHz}$ Wi-Fi. The $900 \mathrm{MHz}$ ISM band is widely used by shorthaul spread-spectrum datalink equipment, and has found wide use in several test management projects over the last few years. Figure 10 shows the upper antenna cluster, while Figure 11 shows the antenna cluster on the lower aft deck of the RASCAL.

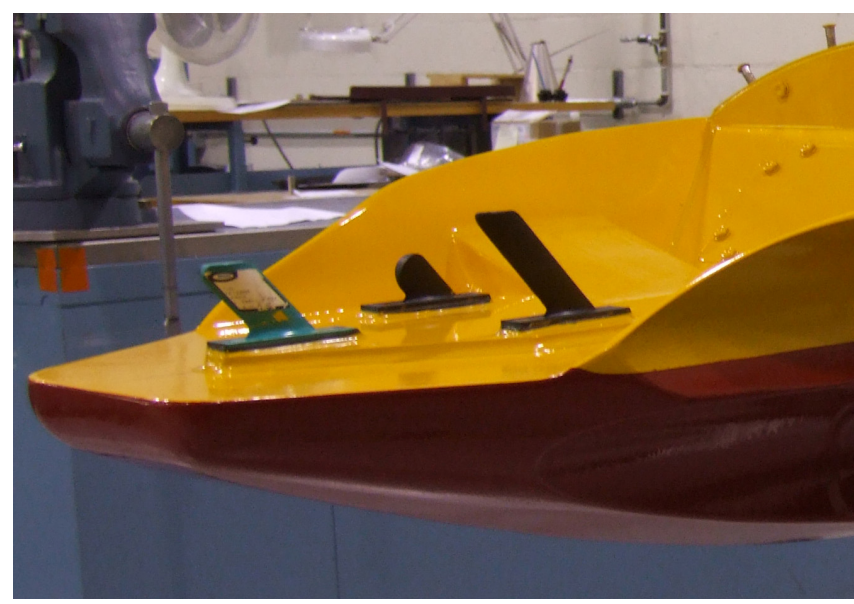

Figure 11 - Rear lower antenna deck showing placement of the $900 \mathrm{MHz}$, L-band, and S-band antennas on the RASCAL. Not shown are diversity antennas for S-band and $900 \mathrm{MHz}$ are located atop the front of the pod, aft of the GPS antenna.

\section{Processor and Data Support Equipment}

Given the ubiquitous nature of the RASCAL, a modular design was desired for the data processing equipment. The PCI Extension for Instrumentation (PXI) [10] provides a highly extensible communication bus standard that has proven reliable and highly compatible between various manufacturers of removable cards. Although several vendors offer PXI card-cages and processor cards, National Instruments [11] manufactures a DC powered mainframe that is compatible with the 24 Volt RASCAL DC power system. Rugged PXI cards are available that span functions such as motion control, RF processing, camera digitizing, 
real-time process control, and high-speed signal processing, to illustrate just a few. To accommodate an 8-slot card cage, the internal width of the RASCAL was expanded to approximately 12.5 inches. It is anticipated that most future projects will require the services provided by the PXI card cage system. Figure 12 illustrates the 8 -slot PXI system that will be installed in the aft equipment bay on a semipermanent basis. Control of the various processor and peripheral cards is simplified by the use of compatible highlevel computing languages such as National Instruments LabView [11] and the Mathworks MATLAB [12].

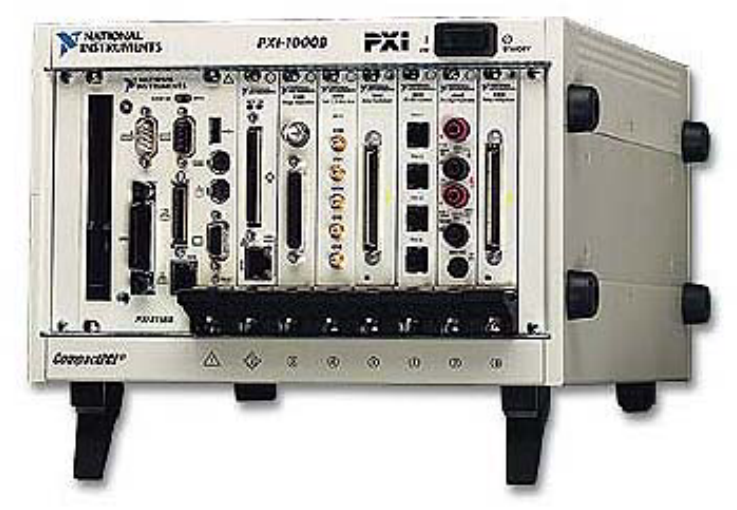

Figure 12 - PXI Processor and signal processing card cage used as data processing equipment. The card cage is installed in a semi-permanent configuration in the aft equipment bay, as it will provide critical processing and data collection functions of future installed sensors.

\section{System Control}

Control of the installed experimental equipment is a primary concern, both by flight worthiness officials, as well as system integrators. It is relatively straightforward to design discrete control signal lines to apply power to individual systems, transport status data, and indicate various functions of the installed experimental equipment. It is quite another matter to route cables to a convenient location within the cockpit for easy access by pilots and test engineers. Fortunately, all production F-16 and AT-38B aircraft have provisions installed to allow electronic control of electronic countermeasure (ECM) pods. Using the pre-existing wiring, remote control of pod equipment power and operating modes is possible without modification of the carriage aircraft.

Additionally, the F-16C/D model aircraft provide for video and MIL-STD-1553 serial data bus connections through standard pylon wiring. Although these connections are intended to convey data and parameters between the aircraft and munitions located on F-16 stations 3 and 7, the wiring makes it convenient to route video and data between the aircraft and RASCAL. For example, standard RS-170
(NTSC) video can be generated by camera and video processing equipment aboard the RASCAL and conveyed to one of the two high-resolution video displays in the front and rear cockpits. Such real-time feedback will be invaluable for training cameras and other sensors at targets of interest during flight. Additionally, MIL-STD-1553 bus access opens up a wide variety of possibilities, including programmatic control of various avionics functions of the host aircraft, as well as RASCAL-based recording of 1553 bus parameters.

Finally, there exists the capability to connect to the pod wirelessly using a wide variety of short-haul Wi-Fi equipment via the S-band antenna provisions of the RASCAL [5]. A compact Wi-Fi enabled computing device carried by a flight test engineer would allow very flexible control and display capability, harnessing the full capacity of processing equipment that might be installed in the RASCAL card cage. This capability transcends limitations imposed by the limited wiring available at the cockpit ECM control panel, although the hardwired control panel is convenient for discrete switching functions that don't require the complexity of a wireless interface.

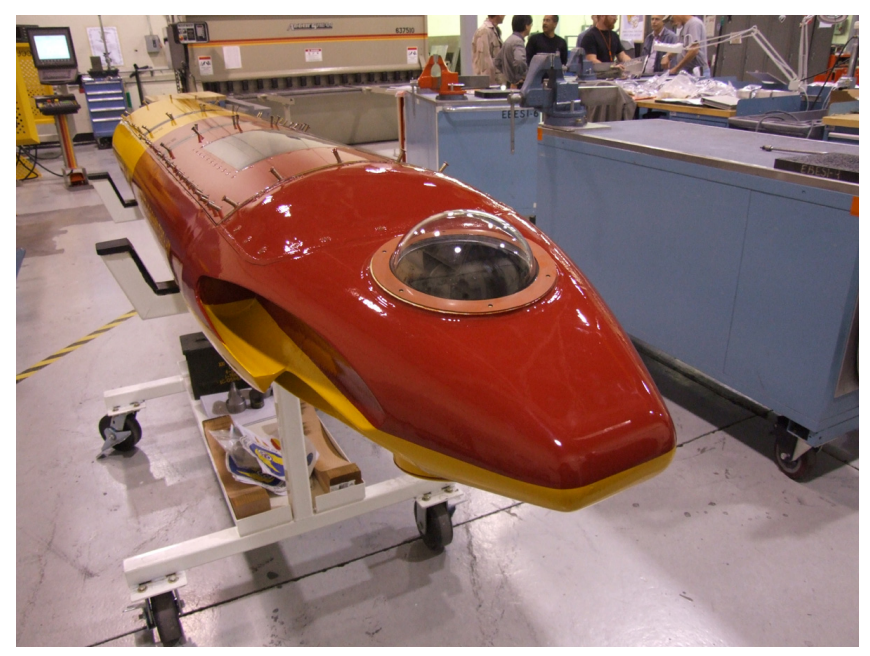

Figure 13 - Overview of the completed RASCAL pod. The pod is shown inverted on a custom roller stand. The hemispherical optical dome has been removed from the opposite surface and capped. Such an arrangement will allow the pod to fly without damage to the domes when not required for a particular test.

\section{Structural AND AERodynamic AnAlysis}

\section{Overview}

The overall goal of the RASCAL modification program is to produce a flight-certified pod capable of housing a wide variety of flight test equipment, to support yet unspecitied TPS test management projects. A supporting goal is to satisfy the regulatory requirements necessary to gain flight 
clearance of the RASCAL on all AFFTC fleet support aircraft. At this stage, the primary focus is RASCAL carriage on all models of the F-16. Subsequent clearance will be investigated on the AT-38B and F-15.

While it is difficult to forecast the exact configurations of future test projects and the associated equipment integrated onto the RASCAL pod, much effort has been spent to make transitions between programs relatively straightforward. For example, the RASCAL team is seeking flight clearance for a baseline configuration that includes the battery/charger equipment, the associated RF and cabling, and worst-case ballast in the forward and aft compartments. The team settled on a maximum requirement of 100 pounds in each the forward and aft equipment bays. It is expected that the vast majority of test projects will require much less than 200 pounds of test equipment.

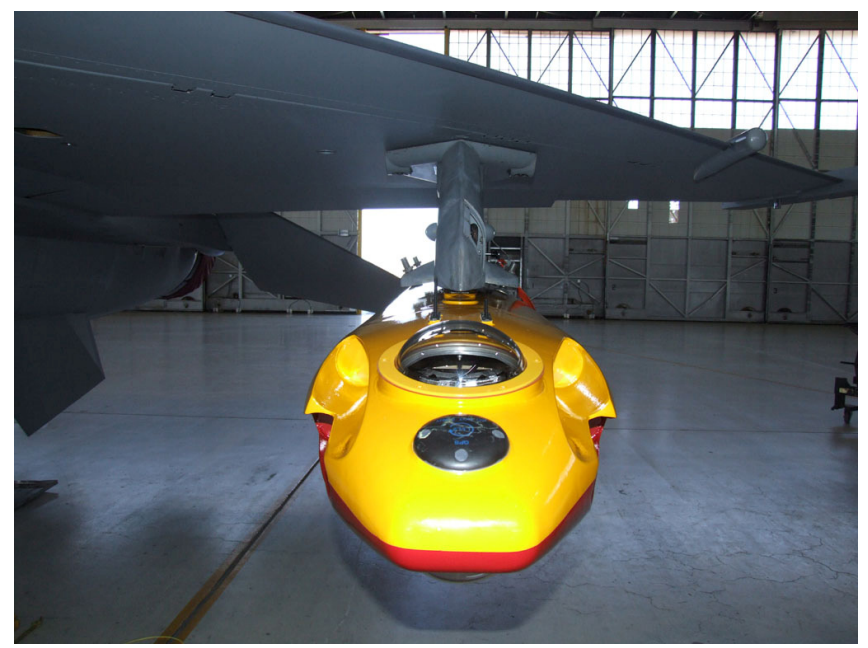

Figure 14 - RASCAL pod viewed from the front under the wing of an F-16D. The circular object forward of the upper dome window is the GPS antenna. The rocket port plugs are visible from this angle.

\section{Structural Model}

A detailed CAD model of the RASCAL was developed using Autocad [13] to facilitate accurate fabrication of all parts, and to conduct a comprehensive finite-element analysis (FEA) of the RASCAL under a series of simulated load conditions. In general, two loading functions are of major concern to flight worthiness officials: loads due to inertia, and loads due to aerodynamic pressures. Inertial loads occur due to the accelerations experienced at dynamic flight conditions such as turns and rolls. Aerodynamic loads change dramatically as a function of airspeed, altitude, and aircraft angle of attack (AOA) and sideslip. The structural modeling software must take into account both factors to accurately predict structural stress failure points. Major stress areas occur at the pylon mounting points where the RASCAL is attached to the aircraft. Figure 15 illustrates an isometric snapshot of the FEA grid used to conduct this analysis. The NASTRAN software package is currently being used to analyze the structural load conditions of the RASCAL.

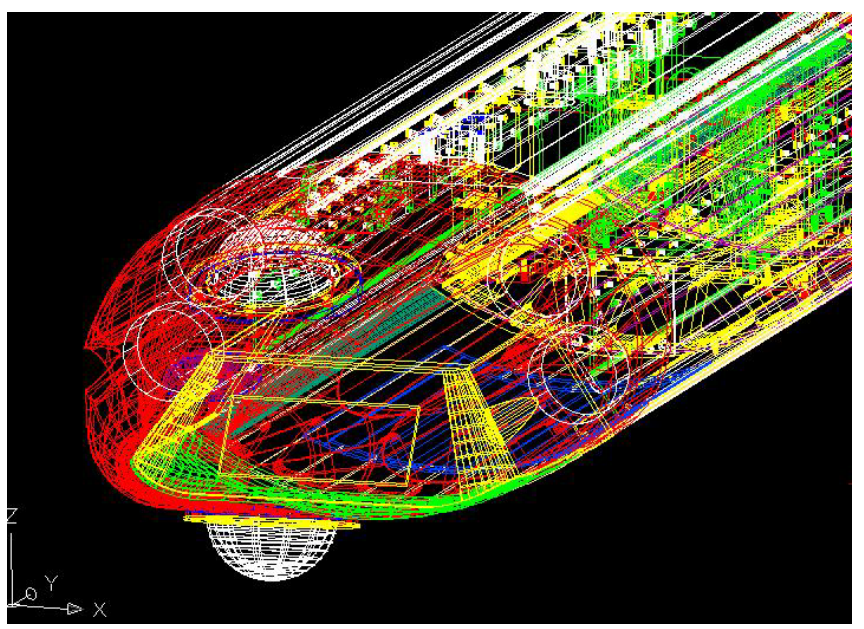

Figure 15 - Grid model used for import into NASTRAN Finite Element Analysis modeling program to determine structural integrity of the RASCAL under various loading and aerodynamic conditions.

\section{Aerodynamic Modeling}

As noted above, the structural model requires accurate aerodynamic loading vectors as input to calculate a comprehensive stress and strain analysis. Several methods exist to accurately predict such data, including analytical, subscale wind-tunnel measurement, and computational methods. The computational fluid dynamic (CFD) method was chosen for this effort because it provides a good balance between accuracy and cost. While wind-tunnel testing provides excellent correlation between actual flight conditions, the cost and schedule expense of such a venture would have been prohibitive. A grid model of the RASCAL was derived from the exterior structural Autocad grid model used for FEA analysis. After error checking the CFD grid using the Gridtool software package developed by NASA Langley Research Center [14], these data are sent to computing clusters of the DoD High Power Computer Center using the Cobalt CFD software package [15]. The Cobalt software allows full Navier/Stokes flow solution which has been found to accurately model dynamic pressures and flow gradients throughout the transonic region and into the supersonic airspeed region. Several data runs are planned at various loading conditions typical of high subsonic, transonic and supersonic speeds. The results of the CFD calculations will be entered as vector fields into the structural FEA model to ascertain the expected stress and strain limits of the pod as a function of airspeed, altitude and relative wind. 


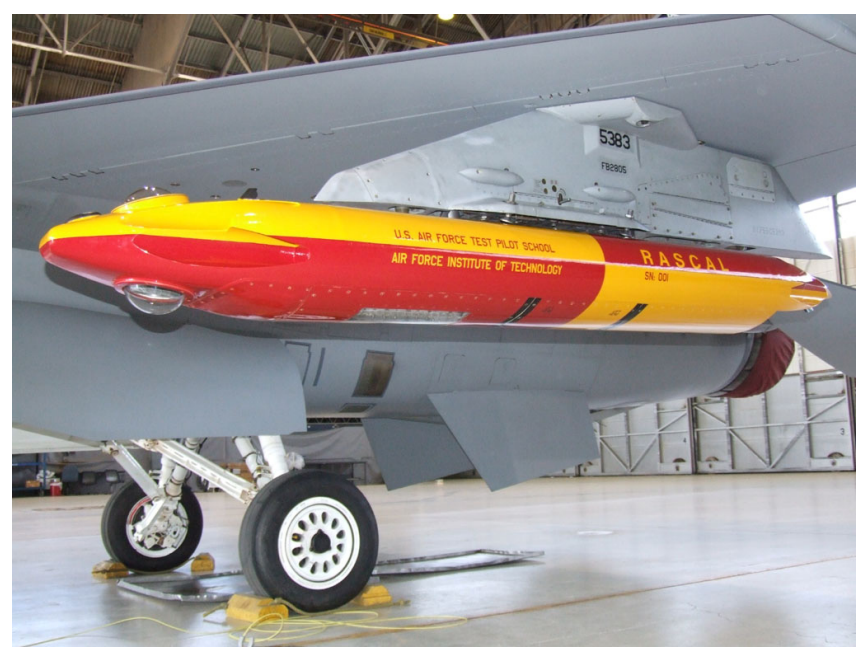

Figure 16 - The completed RASCAL pod under the wing of an F-16D.

\section{Flight Test Plan}

\section{Flight Test Objectives}

Several objectives must be met by the initial flight test program of the RASCAL. The primary objective is to establish flight worthiness of the pod when carried aboard an F-16 aircraft. A secondary flight test objective is to measure the environmental conditions experienced at nominal flight conditions. A final test objective is to validate the structural and aerodynamic models developed to predict safe flight conditions for the pod.

\section{Flight Certification Process}

Initial flight testing will consist of approximately three to four hour-long sorties using an AFFTC fleet support F-16 aircraft. Air Force Seek Eagle Office (AFSEO) at Eglin Air Force Base, Florida, is charged with the mission of managing flight certification testing on all stores and munitions attached to Air Force aircraft. Once AFSEO is satisfied that sufficient structural and aerodynamic analysis has been conducted, a matrix of flight test points will be specified that demonstrates that the store is capable of sustained flight under various expected flight conditions. After successful completion of this series of test points, AFSEO will recommend that flight clearance be granted by the program office responsible for the carriage airframe.

\section{Test Mission Profile}

Example test points include accelerated turns to impart downward stress and strain on the store and pylon attachment hardware, loaded and unloaded rolls at various roll rates to check for lateral stress and strain due to inertial and sideward aerodynamic loads, and high-speed, low altitude runs for extended periods that validate structural integrity under conditions of elevated air loads and increased skin temperatures. Some concern has been raised over the potential for deformation of the hemispherical polycarbonate optical domes and lower optical port due to high air loads under conditions of elevated temperatures that may be encountered at high transonic and supersonic flight. Optically clear polycarbonate is an extremely resilient thermoplastic that has excellent impact resistance over temperatures ranging from $-40^{\circ} \mathrm{F}$ to $280^{\circ} \mathrm{F}$, and has a specified melt point of $310^{\circ} \mathrm{F}$. In order to ascertain the temperature gradations actually experienced in flight, thermocouple transducers will be attached to the inner surface of the upper forward portion of the hemispherical dome, as this is considered a worst-case temperature point due to relatively normal air loads. It was noted during discussions with AFSEO officials that polycarbonate windows have been effectively employed as optical ports on flight test pods at up to Mach 1.5 or greater with no adverse effects such as melting or hazing.

\section{Test Instrumentation}

The RASCAL will be fitted with a variety of flight test instrumentation in order to assist the test team safely explore the operational envelope. In addition to thermocouples placed on the forward optical port, the ambient temperature of the pod interior will be measured throughout the flight test. These data will be extremely useful when determining cooling requirements for heatdissipating electronic payloads such as data processing equipment and related sensors.

In order to safely proceed to high loading and airspeed conditions, select points on the RASCAL surface will be fitted with mechanical strain gauge transducers. The exact location of these transducers is determined by examination of the predicted high-stress points calculated by the structural finite element analysis code. Additionally, tripleaxis accelerometers and acoustic transducers will measure high-bandwidth accelerations and sound pressure levels at several locations within the pod. Analysis of these data will allow future system integrators to manage payload specifications that match the expected operating environment.

Finally, some concern has been raised over the stability of the pod for use in optical measurement research. A visible wavelength camera will be mounted at a forward/downward look angle to capture imagery of opportunity. The resulting recorded video will be analyzed in order to quantify the effects of vibration at various airspeed, Mach and altitude conditions. These data will be correlated with acoustic and accelerometer data to evaluate the required isolation and damping that might be required of optical equipment installed on future experimental test missions.

\section{Real-Time Telemetry of Data}

Given the importance of the measured data to ensuring safe exploration of the pod flight envelope, many of the 
measured parameters will be telemetered to one of two ground station control rooms at the TPS. Figure 17 depicts a typical control room mission in progress at TPS. Standard L-band frequency allocations will be used to send temperature, strain gauge, acceleration and acoustic/vibration data from the RASCAL telemetry transmitter to operator displays in the control room. Simultaneous telemetry data from the aircraft-installed data acquisition system will present airspeed, altitude, mach, and aircraft loading data to the same control room via an independent L-band channel. The real-time correlation of flight condition with pod characteristics will allow a disciplined and safe transition to test points that carry enhanced risk due to loading and airspeed.

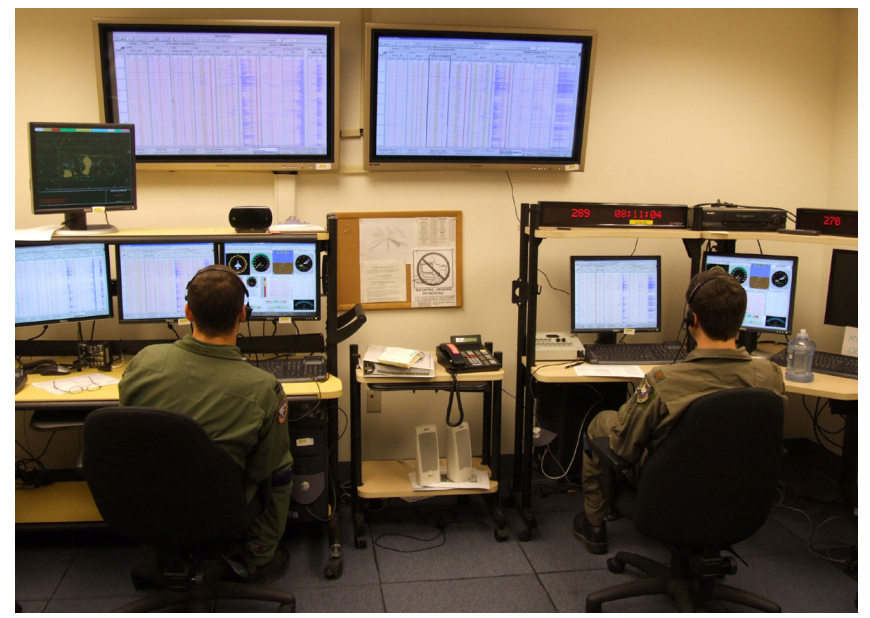

Figure 17 - One of two identical mission control rooms at the USAF Test Pilot School. Electronic strip charts plot time history of a wide variety of parameters telemetered from both the RASCAL pod and the F-16 data acquisition system.

\section{CONCEPT OF OPERATIONS}

The RASCAL described in the previous sections is the prototype of a series of four such devices to be built immediately following successful flight test certification. After an initial series of acceptance tests, three additional RASCAL pods will be concurrently built by the instrumentation division of the $412^{\text {th }}$ Test Wing at Edwards Air Force Base. Since a large fraction of future development and integration work will take place at AFIT or AFRL, a cycle will be developed whereby two pods are readied for flight test at Edwards, while the remaining two pods undergo development at remote locations such as AFIT or one of the National Laboratories. This concept will allow longer lead development and reduce the amount of travel required to integrate complex equipment onto a particular pod within prohibitive time constraints. It is anticipated that the remaining pods will be built by the end of the 2008 fiscal year.
Once an experiment has been conceptualized, the idea will take form via preliminary and critical design review teams. After acceptance, the experimental hardware will be loaded and inspected. The overall system will undergo weight and balance testing to ensure that load limits have not been exceeded, and a procedure for conducting electromagnetic compatibility and interference mitigation (EMC/EMI) checks will be finalized. A detailed procedure developed by officials at the USAF TPS will be followed to ensure that final checks and quality assurance by AFFTC officials is fully complied with. In general, external modifications will require elevated oversight by AFFTC and $412^{\text {th }}$ Test Wing officials.

\section{LASER RAdar EXaMPle PAYload}

\section{3-D Laser Radar Experimental Research}

Following the initial certification flight test planned for late Fall 2007, work will begin on integration of a novel Flash Laser Radar (LADAR) onto RASCAL in order to conduct risk-reduction data collection and flight test for a series of future AFIT/TPS test management projects.

AFIT faculty and students, together with scientists and engineers at the Air Force Research Laboratory Sensors Division (AFRL/SN) have great interest in furthering the state-of-the art of current laser sensors [16], [17], [18]. AFIT, TPS and AFRL have partnered to launch a series of LADAR data collection missions to generate a large database of experimental data to validate analytical and simulated models, as well as spawn new research in 3-dimensional LADAR imagery. The imagery collected will enable quantification of fundamental 3-D laser radar performance limitations, development of novel image processing and calibration algorithms, and exploration of operational limitations and concerns surrounding implementation of such a device in an operationally representative dynamic flight environment.

3-D Flash LADAR [19] is an exciting new development. Although scanning 3-D laser radar systems have been implemented for quite some time, Flash LADAR develops an entire image array in a single instant across a fairly large imaging array. Current state-of-the-art limits array sizes to $128 \times 128$ elements, large enough for very detailed imagery given suitable optical fields-of-view. Frame rates are quite fast, typically up to 30 frames-per-second given adequate cooling capability of the gated laser illuminator. Figure 18 shows a commercial Flash LADAR system developed by Advanced Scientific Concepts (ASC) of Santa Barbara California [20]. 


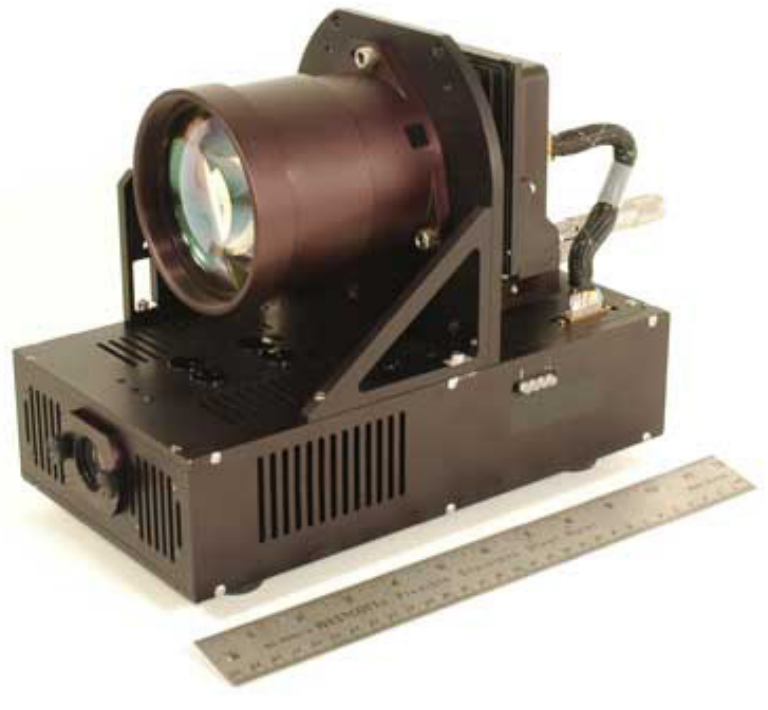

Figure 18 - Flash Laser Radar (LADAR) manufactured by Advanced Scientific Concepts of Santa Barbara California. The lower portion is an eyesafe laser illuminator with a pulse repetition period synchronized to the camera gate. The actual camera is quite small, located directly behind the optical lens.

The compact size of the ASC Flash LADAR allows for straightforward integration into the prototype RASCAL pod through downward angled optical paths. Analysis shows that look angles depressed 20 or more degrees below the aircraft waterline are achievable without great difficulty. Rearward camera mounting allows forward viewing through a primary surface mirror. Such a simple, fixed optical system would provide limited tracking ability of ground or airborne targets, but would greatly simplify system mechanization. Gimbal of the mirror in at least one axis would allow more flexible imaging at the expense of increased system complexity. Figure 19 shows a CAD rendition of the proposed RASCAL LADAR installation.

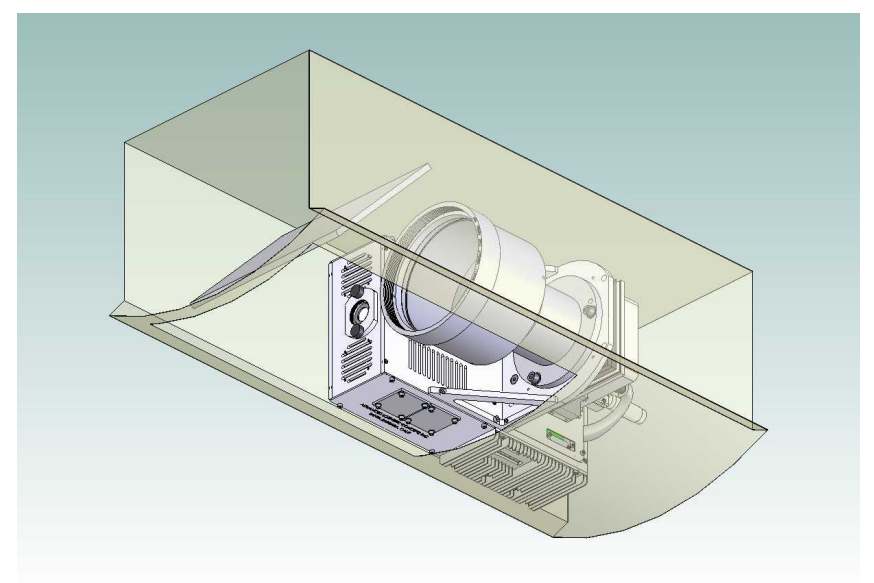

Figure 19 - CAD rendering of Flash LADAR installed in RASCAL forward equipment bay. The optics are pointed aft in the pod to allow the primary surface mirror to reflect for a forward/downward viewing angle. The $250 \mathrm{~mm}$ receiver lens will allow optical survey to ranges exceeding 2 kilometers.

RASCAL Flash LADAR data collection flight tests are scheduled to begin in May 2008 in support of September 2009 test management projects. A variety of ground and air target imagery will be collected under a wide range of optical conditions.

\section{Conclusion}

This paper provided an overview of the concept, design, analysis, planned flight test, and future employment of a reconfigurable flight test research pod. The pod has been carefully engineered to allow introduction of novel flight hardware without costly and time-consuming modification to flight test aircraft. Detailed structural and aerodynamic models constructed for the RASCAL will allow future modifications to be conducted with minimal manpower. The RASCAL will allow future scientists, engineers and flight test students to rapidly transition cutting-edge technology to the physically demanding flight environment with minimal cost and schedule burden on all agencies involved.

Although much has been accomplished on the RASCAL project, several milestones need to be reached in order to fully realize the utility of the system. The actual flight test of the RASCAL prototype needs to be executed and data collected to understand any limitations or unknown capabilities inherent in the pod design. Three additional RASCAL pods need to be constructed and flight tested. A streamlined operational employment process is under development to allow rapid replacement of internal hardware and test equipment. Finally, AFIT and TPS teams need to put forth exciting and rewarding projects for integration onto the RASCAL fleet.

The author would like to thank the efforts of Dr Stephen Cain for providing initial funding for concept exploration of the RASCAL. Additional funding has been the AFIT office of engineering research and the AFIT Advanced Navigation and Technology laboratory. The tremendous support offered by the USAF TPS leadership and the TPS Plans and Programs division have made the continued funding of this project a reality. Finally, great credit must be given to the outstanding $412^{\text {th }}$ Test Wing RASCAL team, comprised of design engineers, model-makers and program support personnel. The opinions and views expressed by the author are not necessarily those of the Department of Defense or the United States Air Force. 


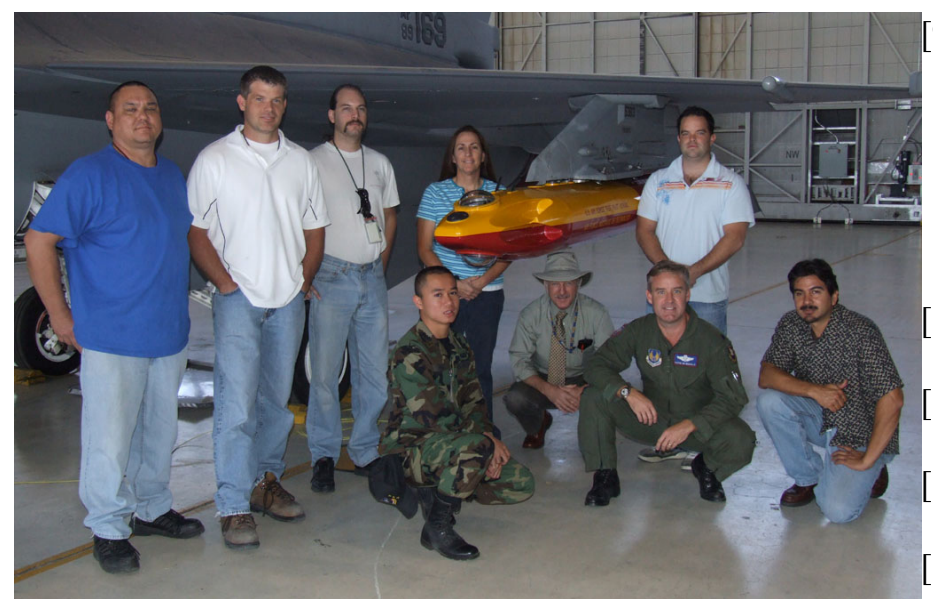

Figure 20 - The RASCAL design team under the wing of an AFFTC F-16D.

\section{REFERENCES}

[1] U.S. Air Force Test Pilot School web site http://www.edwards.af.mil/library/factsheets/factsheet.asp $? \mathrm{id}=6467$

[2] D. Gleason, D. Walker and N. Howell, "USAF Test Pilot School Test Management Program," Aerospace Sciences Meeting and Exhibit, 34th, Reno, NV, AIAA-1996-159, Jan. 15-18, 1996

[3] Air Force Flight Test Center at Edwards Air Force Base California web site http://www.edwards.af.mil/

[4] Air Force Institute of Technology, Dayton, Ohio web site http://www.afit.edu

[5] A. MacDonald, "Low-Power Wireless Local Area Networks for Flight Test," 2007 IEEE Aerospace Conference Proceedings, \#1371, March 1-8, 2007.

[6] M. Nielsen, C. Bradley, D. Itsara, W. Grac, L. Viarengo, and B. Pacer, "Limited Evaluation of the SLAMMR Inertial Optical Sensor Fusion Algorithm (Project GOOD LOOKIN'), US Air Force Test Pilot School, August 2007.

[7] J. Raquet and M. Giebner, "Navigation Using Optical Measurements of Objects at Unknown Locations," in Proceedings of the 59th Annual Meeting of the Institute of Navigation, pp. 282-290, June 2003.

[8] M. Veth and J. Raquet, "Alignment and Calibration of Optical and Inertial Sensors Using Stellar Observations," US Air Force Institute of Technology, Tech Report no. A869264, June 2007.
9] M. Starr, G. Reboul, J. Hasker, C. Molina, R. Reichart, H. Hall and M. Lampley, "Limited evaluation of a direct injection embedded jamming system in the T-38C (Project TOE JAM)," Air Force Flight Test Center Technical information Memorandum, AFFTC-TIM-05-09, June 2005 .

[10] PXI Systems Alliance website http://www.pxisa.org/

11] National Instruments website http://www.ni.com

[12] Mathworks website http://www.mathworks.com

[13] Autodesk website http://www.autodesk.com

[14] NASA Langley Research Center Geometry Laboratory website http://geolab.larc.nasa.gov/GridTool/

[15] Cobalt Solutions Computational Fluid Dynamics web site http://www.cobaltcfd.com/index.php/site/

[16] A. MacDonald and S. Cain, "Maximum a posteriori image and seeing condition estimation from partially coherent two-dimensional light detection and ranging images," Optical Engineering, vol. 45, no. 8, Aug. 2006.

[17] A. MacDonald and S. Cain, "Parameterized blind deconvolution of laser radar imagery using an anisoplanatic OTF," Optical Engineering, vol. 45, no. 11, Nov. 2006.

[18] S. Cain, R. Richmond, and E. Armstrong, "Flash light detection and ranging range accuracy limits for returns from single opaque surfaces via Cramer-Rao bounds" Applied Optics, Vol. 45, Issue 24, pp. 6154-6162.

[19] R. Stettner, H. Bailey and R. Richmond, "Eye Safe Laser Radar Focal Plane Array for Three-Dimensional Imaging," Proceedings of the SPIE Vol. 5412; laser radar technology and applications IX, April 14, 2004.

[20] Advanced Scientific Concepts website http://www.advancedscientificconcepts.com/index.html 


\section{BIOGRAPHIES}

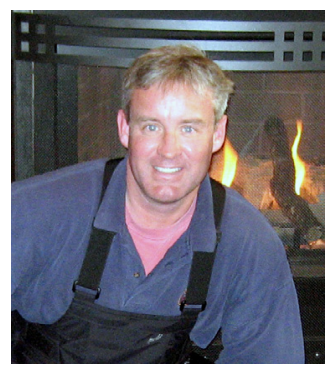

Adam MacDonald received the B.S.E.E. degree in electrical engineering from the University of Massachusetts in 1988 and M.S.E.E. and Ph.D degrees in electrical engineering from the Air Force Institute of Technology (AFIT) in 1999 and 2006 respectively. He gained his Air Force commission in 1988 and flew the F-15C as an operational fighter pilot and as an experimental test pilot. A graduate of the Air Force Test Pilot School, Lieutenant Colonel MacDonald has flown over 30 different aircraft and has accumulated over 2000 flight hours in fighter-type aircraft. He has worked on diverse developmental test programs such as Laser Radar, AIM-9X, Combat-ID, Fighter Data-Link, and avionics modernization for the T38C. He is currently assigned as Director of Education and Instructor Test Pilot at the US Air Force Test Pilot School, Edwards Air Force Base, California, and is an Adjunct Professor at AFIT.

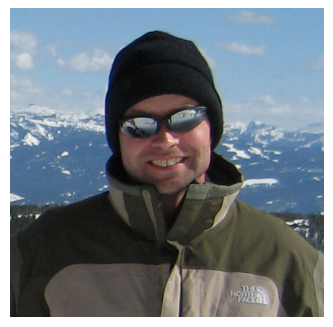

Michael Shepherd is the Director of Plans and Programs, United States Air Force Test Pilot School. Lieutenant Colonel Shepherd's research interests include structural dynamics of aerospace structures and flight test. Lt Col Shepherd obtained a B.S. from the USAF Academy, an M.S. from the University of Washington, and a Ph.D. from the Air Force Institute of Technology. He is a master navigator with over 3000 flight hours, a graduate of the USAF Test Pilot School and a member

AIAA. 